\title{
Identify the radiotherapy-induced abnormal changes in the patients with nasopharyngeal carcinoma
}

\author{
Xiuxin Wang ${ }^{1+}$, Yi Zhao $^{1+}$, Huiling Zhang ${ }^{1}$, Gang Yin ${ }^{2}$, Yangkun Luo $^{2}$, Zixuan Fan ${ }^{2}$, Yin \\ $\operatorname{Tian}^{1 *}$ \\ ${ }^{1}$ Bio-information College, Chong Qing University of Posts and Telecommunications, Chong Qing, China \\ ${ }^{2}$ Department of Radiotherapy, Sichuan Cancer Hospital \& Institute, Chengdu, China
}

*Corresponding Author: E-mail: tiany20032003@163.com; Tel.: +86-23-62460536; Fax: +86-23-62460536

+ The authors have equal contribution to this work

Received: December 02, 2017; Revised: December 12, 2017; Published: December 24, 2017

\begin{abstract}
Radiotherapy (RT) is the standard treatment for nasopharyngeal carcinoma, which often causes inevitable brain injury in the process of treatment. The majority of patients has no abnormal signal or density change of the conventional magnetic resonance imaging (MRI) and computed tomography (CT) examination in the long-term follow-up after radiation therapy. However, when there is a visible CT and conventional MR imaging changes, the damage often has been severe and lack of effective treatments, seriously influencing the prognosis of patients. Therefore, the present study aimed to investigate the abnormal changes in nasopharyngeal carcinoma (NPC) patients after RT. In the present study, we exploited the machine learning framework which contained two parts: feature extraction and classification to automatically detect the brain injury. Our results showed that the method could effectively identify the abnormal regions reduced by radiotherapy. The highest classification accuracy was $82.5 \%$ in the abnormal brain regions. The parahippocampal gyrus was the highest accuracy region, which suggested that the parahippocampal gyrus could be most sensitive to radiotherapy and involved in the pathogenesis of radiotherapy-induced brain injury in NPC patients.
\end{abstract}

\section{Keywords}

nasopharyngeal carcinoma (NPC); radiotherapy (RT); injury; classification; structural MRI (sMRI);

\section{Introduction}

Nasopharyngeal carcinoma (NPC) is an endemic disease and rare in most regions of the world. However, it exists much more frequently in Southeast Asia and China. In Southern China, NPC is one of the most common malignant tumor and the incidence of NPC is approximately 30-80 per 100,000 per year [1]. Because of the special lesion location, nasopharyngeal carcinoma is not suitable for surgical treatment and radiotherapy plays a crucial part in NPC therapy. However, brain injury caused by radiation therapy is a serious complication, which has a serious impact on the prognosis and quality of life of the patients. Thus, it is necessary to understand the effect of radiation therapy on brain structure and find neural biomarkers to facilitate clinical diagnose, treatment, and prevention [2].

Previous studies have found that radiation therapy for NPC resulted in multiple regions abnormalities, 
such as temporal lobe necrosis [3, 4], precuneus, cuneus, lateral occipital cortex [5], the vermis, hippocampus, cerebellum lobule, middle occipital lobe, and insula [6,7]. However, it was difficult for doctors to identify the abnormal changes after radiotherapy (RT) based on original image features. There was a lack of automatic classification method for NPC patients before and after RT based on the big data of sMRI. Therefore, we hoped that the machine learning framework with sMRI could improve the present situation on brain radiation therapy.

\section{Materials and Methods}

\section{Participants}

The total participants included 103 right-handed NPC patients ( 80 males and 23 females, mean \pm SD $=$ $47.5 \pm 10.8$ years, age from 23 to 82 years). All the patients were recruited from the Sichuan Cancer Hospital in Chengdu, China, and they all had no other disease. Each subject performed two MRI scans, i.e. before (pre-RT) and after radiotherapy (post-RT).

The research was approved by the Ethics committee of Sichuan Cancer Hospital \& Institute, Chengdu, China. Written informed consent was obtained from all subjects.

\section{MRI acquisition}

The sMRI images of the whole brain were obtained by the rapid acquisition of T1 weighted sequences with a high-resolution three-dimensional magnetic Spin echo using a Siemens $1.5 \mathrm{MR}$ scanner (TR $=750$ $\mathrm{ms}, \mathrm{TE}=11 \mathrm{~ms}, \mathrm{FOV}=75 \times 75 \mathrm{~cm}$, flip angle $=150^{\circ}$, matrix size $=256 \times 192$, voxel size $=1 \times 1 \times 1 \mathrm{~mm}^{3}$, slice thickness $=3 \mathrm{~mm}$, layer space $=3.6 \mathrm{~mm}$, the continuous acquisition $=36$ layers covering the whole brain .

The voxel-based morphometry (VBM) toolbox of the Statistical Parametric Mapping software (SPM8) was used to perform the data-preprocessing. Firstly, the structured images were registered into the standard MNI space according to 12 radiation parameters. Secondly, the spatially normalized images were segmented into gray matter, white matter and cerebrospinal fluid using a priori tissue probability maps (TPM) provided by International Consortium for Brain Mapping (ICBM). Thirdly, the segmented gray images were further smoothed with a kernel of $8 \mathrm{~mm}$ to remove the influence of noise and increase the validity of statistical tests of the posterior parameters.

\section{Methods}

The methods mainly included two parts: 1) feature extraction and feature selection with the principal component analysis (PCA); 2) classification with support vector machine (SVM). Details were shown in Figure1. A $3 \times 3 \times 3$ cube was defined and vectorized. Leave-one-out cross-validation (LOOCV) was used to evaluate the general classification performance. Image data were divided into two sessions: one was a test session whose size were $1 \times 27$ was leaving out by turns in each round of LOOCV, and the other was a training session whose size were $205 \times 27$. Then, PCA was used to extract the features of the training session. LIBLINEAR was an open source library for large-scale linear classification that inherits many features of the popular SVM library LIBSVM. In this paper, we used the LIBLINEAR toolbox [8] to perform the classification task. 


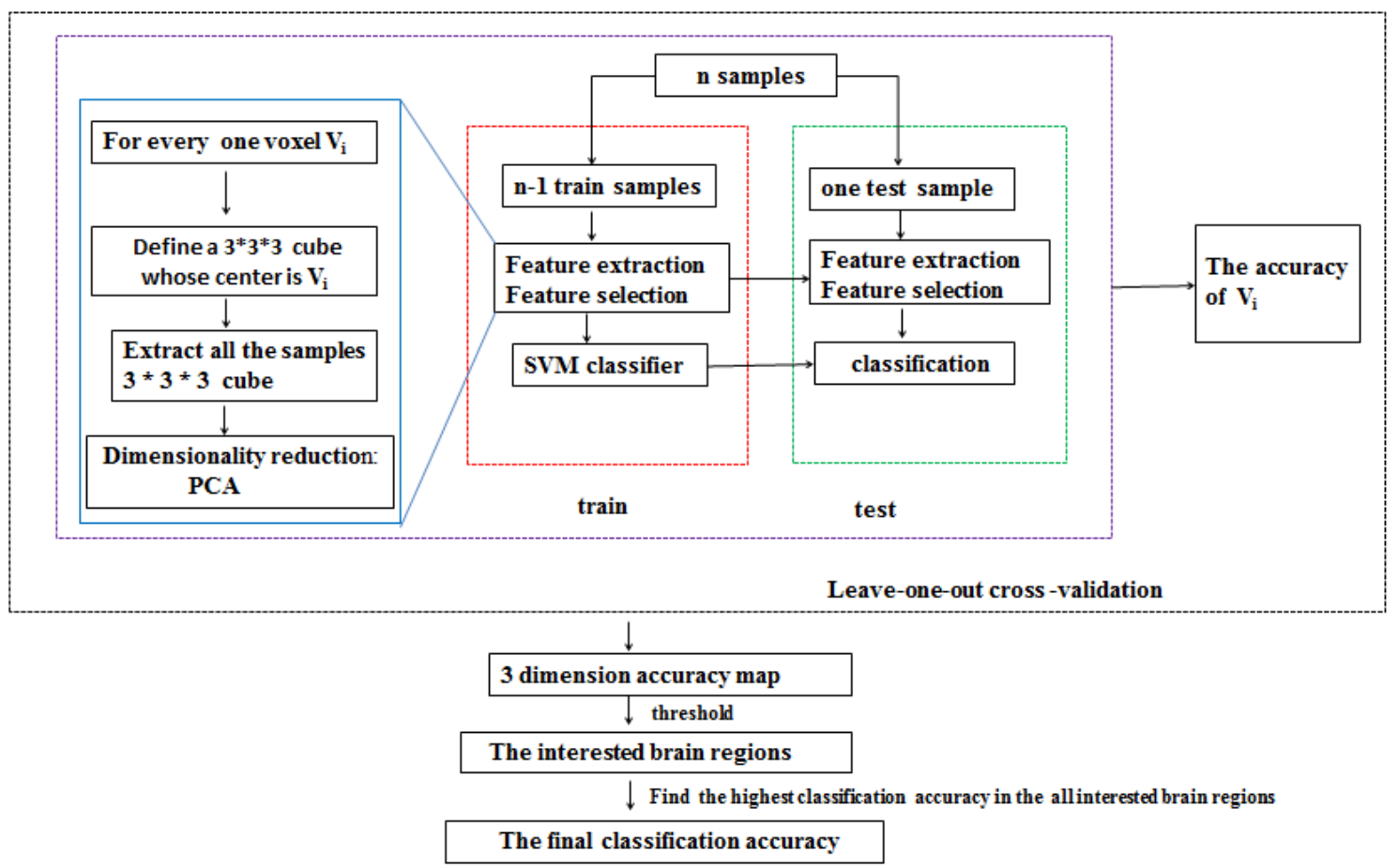

Figure1. The flow chart of classification. $\mathrm{V}_{\mathrm{i}}$ : voxel $\mathrm{i}$; $\mathrm{n}$ was the number of patients; PCA: principal component analysis.

\section{Results and Discussion}

As shown in Figure 2, the final accuracy for distinguishing the pre-RT and post-RT was $82.5 \%$. The interested brain regions should be met: 1 ) the clusters must contain more than 50 continuous voxels. 2) The accuracy of all the voxels in the cluster must be greater than $70 \%$. The final classification accuracy was the greatest accuracy of the voxels in all the interested brain clusters, and the regions in the interested brain clusters were the most distinguishable brain regions. In this study, we identified some interested brain regions which mainly included the bilateral parahippocamps, the bilateral middle temporal gyrus, the right middle occipital gyrus, the left rolandic operculum, the bilateral cerebellum, the vermis, the right hippocampus, the right thalamus, the insula, the right precuneus, and the left cuneus. Details were shown in the Table 1.

In this study, we used the machine learning framework based on feature extraction and classification to achieve the automatic classification between the pre-RT and post-RT. The method of PCA+SVM was quite helpful to improve the accuracy of the classification, and validly detected the abnormal brain regions, which widely contained the previous research achievement. As shown in Table 1, our results were consistent with previous studies, suggesting that the method was in favour of identifying the injury caused by RT [5-7, 9]. The classification accuracy was $82.5 \%$, indicating that the method could well identify the abnormal changes induced by RT. The abnormal brain regions can be considered as biomarkers to detect the radiation-induced injury and help doctors diagnose and monitor the disease.

Some researchers revealed that the parahippocampal cortex and hippocampus were involved in memory [10-13]. The parahippocampal cortex played a role for spatial configuration of object in memory [14]. Hippocampus was associated with attention processes and visuospatial working memory [15]. The temporal lobe necrosis was the most debilitating late-stage complication[16]. Cui et al [17] found that the temporal lobe played critical roles in auditory and language processing, which might be associated with auditory verbal hallucinations (AVHs) for schizophrenia patients. Temporal regions might also be 


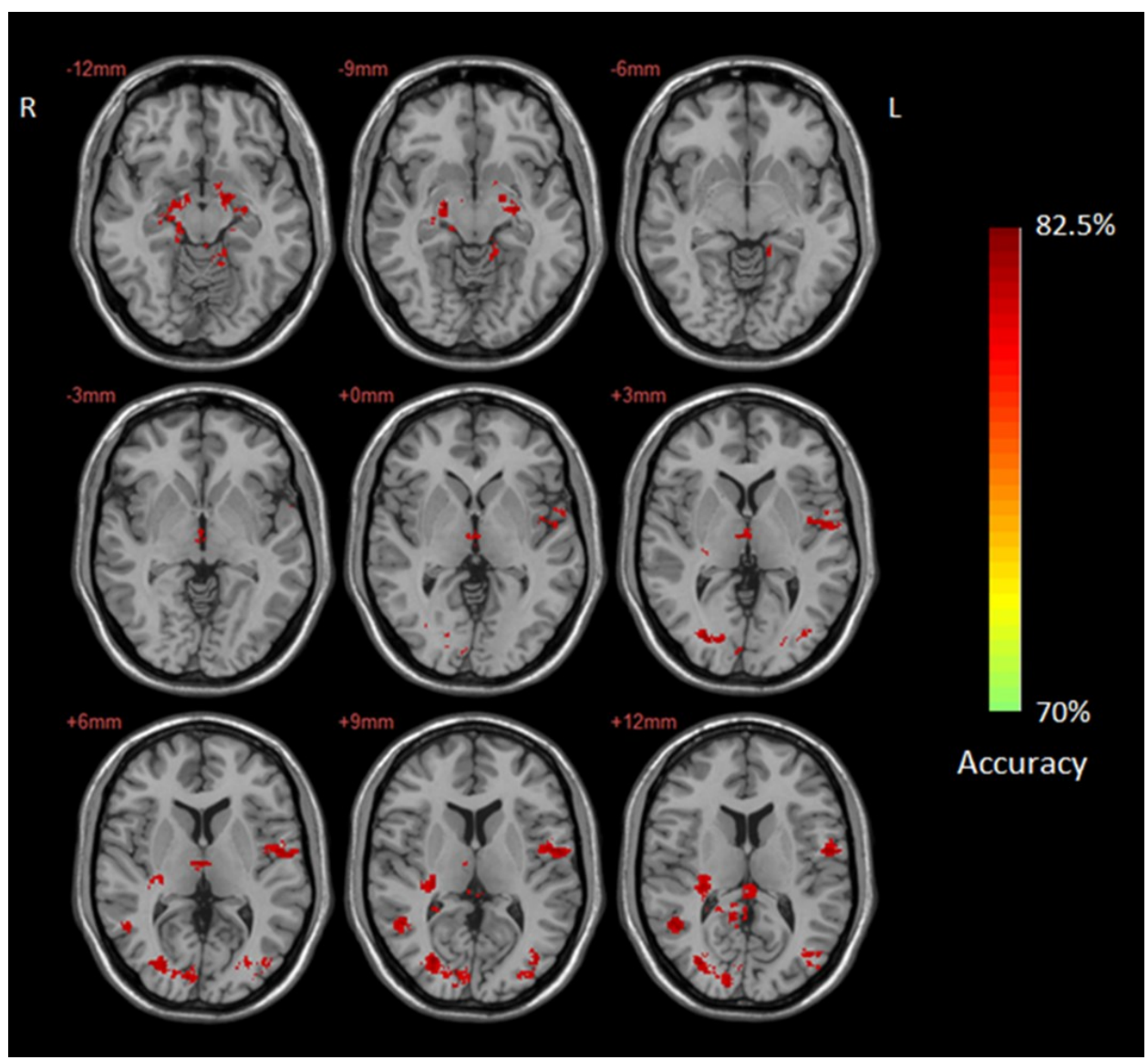

Figure 2. The classification accuracies of NPC patients before RT and after RT based on the PCA and SVM

Table 1. The most distinguish force of gray matter volume brain regions for the pre-RT and post-RT groups

\begin{tabular}{lccccc}
\hline & & \multicolumn{3}{c}{ Peak coordinates } & \\
\cline { 2 - 4 } Clusters & Voxels & $\mathrm{X}$ & $\mathrm{y}$ & $\mathrm{Z}$ & Accuracy of the peak \\
\hline ParaHippocampal_L & 575 & -16.5 & -19.5 & -24 & $82.5 \%$ \\
Temporal_Mid_R & 186 & 48 & -54 & 13.5 & $80 \%$ \\
Occipital_Mid_R & 996 & 28.5 & -78 & 10.5 & $80 \%$ \\
Rolandic_Oper_L & 496 & -57 & -6 & 9 & $77.5 \%$ \\
Cerebellum_9_L & 177 & -12 & -45 & -51 & $77.5 \%$ \\
Cerebellum_9_R & 129 & 7.5 & -51 & -40.5 & $75 \%$ \\
Vermis_1_2 & 716 & 1.5 & -40.5 & -22.5 & $80 \%$ \\
Hippocampus_R & 183 & 19.5 & -10.5 & -9 & $80 \%$ \\
ParaHippocampal_R & 60 & 18 & -24 & -15 & $75 \%$ \\
Thalamus_R & 137 & 4.5 & -13.5 & 4.5 & $75 \%$ \\
Temporal_Mid_L & 480 & -45 & -70.5 & 18 & $77.5 \%$ \\
Insula_R & 723 & 30 & -28.5 & 12 & $77.5 \%$ \\
Precuneus_R & 495 & 9 & -51 & 13.5 & $77.5 \%$ \\
Cuneus_L & 61 & -13.5 & -57 & 18 & $75 \%$ \\
\hline
\end{tabular}

Ps. ParaHippocampal_L: left parahippocampal gyrus; Temporal_Mid_R: right middle temporal gyrus; Occipital_Mid_R: right middle occipital gyrus; Rolandic_oper_L: left rolandic operculum; Cerebellum_9_L: left cerebellum; Cerebellum_9_R: right cerebellum; Hippocampus_R: right hippocampus gyrus; ParaHippocampal_R: right paraHippocampal gyrus; Thalamus_R: right thalam; Temporal_Mid_L: left middle temporal gyrus; Insula_R: right insula; Precuneus_R: right precuneus; Cuneus_L: left cuneus. 
involved in the Human understanding insight which occupied an important position in understanding the clinical heterogeneity of obsessive-compulsive disorder (OCD) [18]. Glasser and Rilling [19] found that the temporal lobe, especially the left superior and middle temporal gyri, was a pivotal part of language pathway in the brain's language network.

The cerebellum was vital to the motor control, cognitive and affective regulation [7]. Shen et al. [20] reported that the cerebral microbleeds (CMBs) observed in the cerebellum were related to cognitive dysfunction.

The insula participated in numerous cognitive processing, including goal-directed cognition, conscious awareness, autonomic regulation, interoception and somatosensation [21].

The precuneus was found to be connected with a wide range of cognitive processes, involving reflective and self-related processing [22, 23], awareness and conscious information processing [24, 25], episodic memory [26-28], and visuospatial processing [29, 30].

The cuneus was significantly involved with visual processing [30, 31]. Collignon et al. [32] demonstrated that auditory-spatial processing mainly recruited the right cuneus and the right middle occipital gyrus, and the specific occipital regions in visuospatial / motion processing for sighted individuals.

The thalamus was believed to regulate and coordinate cortical activity both within and across functional regions, such as motor and visual cortices [33].

Wu et al. [34] found that the NPC patients after radiotherapy demonstrated significant changes in cognition which mainly referred to short term memory, delayed recall, language, attention, orientation, visuo-spatial and executive function. In addition, the patients showed a relatively high rate of general intelligence impairment, which might be related to a longer post-RT interval of mean 4.3 years and bigger total dosage of mean 70.7Gy. Tang et al [35] reported that NPC patients with radiation-induced brain injury (RI) exhibited negative emotions, impaired cognitive function and quality of life (QOL). The accumulated studies showed that the radiation-induced functional impairments included the disorder of short-term memory [36], personality changes [37] and motor abilities [38], a marked anterograde memory impairment for verbal material [39], neuropsychological impairments in recent memory, immediate and delayed verbal recall, and immediate visual recall [40].

Hence, we inferred that these abnormal brain regions might be correlated with the dysfunction of patients with NPC after radiotherapy, which might reveal a potential mechanism for radiation-induced impairment. Furthermore, it was clear that the machine learning framework proposed in the current study could provide an effective reference for identifying the brain injury induced by radiotherapy in clinical diagnosis.

\section{Conclusions}

In summary, this present study proposed an automatic classification method for pre-RT and post-RT. The experimental results showed that the method could effectively identify the abnormal changes between pre-RT and post-RT. Furthermore, these changes might be considered as an underlying biomarker to detect the radiation-induced impairment. 
Acknowledgements: This research is supported by the Chongqing Research Program of Basic Science and Frontier Technology (No. cstc2017jcyjBX0007; No. cstc2015jcyjA10024).

\section{References}

[1] L.L. Tang, R. Guo, G. Zhou, Y. Sun, L.Z. Liu, A.H. Lin, H. Mai, J. Shao, L. Li, J. Ma. Prognostic value and staging classification of retropharyngeal lymph node metastasis in nasopharyngeal carcinoma patients treated with intensity-modulated radiotherapy. Plos One 9 (2014) e108375.

[2] C. Li, Y. Gang. An overview on the role of telomere, telomerase in degenerative diseases and cancer. Admet \& Dmpk 3 (2015).

[3] A.T. Chan. Nasopharyngeal carcinoma. Annals of Oncology Official Journal of the European Society for Medical Oncology 21 Suppl 7 (2010) vii308.

[4] S. Ying, G.Q. Zhou, Z.Y. Qi, Z. Li, S.M. Huang, L.Z. Liu, L. Li, A.H. Lin, J. Ma. Radiation-induced temporal lobe injury after intensity modulated radiotherapy in nasopharyngeal carcinoma patients: a dosevolume-outcome analysis. BMC Cancer, 13,1(2013-08-27) 13 (2013) 397-397.

[5] J. Lin, X. Lv, M. Niu, L. Liu, J. Chen, F. Xie, M. Zhong, S. Qiu, L. Li, R. Huang. Radiation-induced abnormal cortical thickness in patients with nasopharyngeal carcinoma after radiotherapy. Neuroimage Clinical 14 (2017) 610-621.

[6] Q. Ma, D. Wu, L.L. Zeng, H. Shen, D. Hu, S. Qiu. Radiation-induced functional connectivity alterations in nasopharyngeal carcinoma patients with radiotherapy. Medicine 95 (2016) e4275.

[7] Q. Ma, L.L. Zeng, J. Qin, Z. Luo, J. Su, D. Wu, S. Qiu, D. Hu. Radiation-induced cerebellar-cerebral functional connectivity alterations in nasopharyngeal carcinoma patients. Neuroreport 28 (2017) 705.

[8] R.E. Fan, K.W. Chang, C.J. Hsieh, X.R. Wang, C.J. Lin. LIBLINEAR: A Library for Large Linear Classification. Journal of Machine Learning Research 9 (2008) 1871-1874.

[9] X.F. Lv, X.L. Zheng, W.D. Zhang, L.Z. Liu, Y.M. Zhang, M.Y. Chen, L. Li. Radiation-induced changes in normal-appearing gray matter in patients with nasopharyngeal carcinoma: a magnetic resonance imaging voxel-based morphometry study. Neuroradiology 56 (2014) 423-430.

[10] Y. Tian, H. Zhang, W. Xu, H. Zhang, L. Yang, S. Zheng, Y. Shi. Spectral Entropy Can Predict Changes of Working Memory Performance Reduced by Short-Time Training in the Delayed-Match-to-Sample Task. Frontiers in Human Neuroscience 11 (2017) 437.

[11] Y. Tian, Z. Ding, K.Y. Tam, Z. Wang, H. Zhang, D. Zhao, Y. Zhao, W. Xu, S. Zheng. Specific frequency bands of amplitude low-frequency fluctuations in memory-related cognitive impairment: predicting Alzheimer's disease. ADMET \& DMPK 3 (2015) 659-668.

[12] Y. Tian, L. Yang, S. Chen, D. Guo, Z. Ding, K.Y. Tam, D. Yao. Causal interactions in resting-state networks predict perceived loneliness. Plos One 12 (2017) e0177443.

[13] Y. Tian, L. Yang, W. Xu, H. Zhang. Predictors for drug effects with brain disease: Shed new light from EEG parameters to brain connectomics. European Journal of Pharmaceutical Sciences 110 (2017) 2636.

[14] V.D. Bohbot, J.J.B. Allen, A. Dagher, S.O. Dumoulin, A.C. Evans, M. Petrides, M. Kalina, K. Stepankova, L. Nadel. Role of the parahippocampal cortex in memory for the configuration but not the identity of objects: converging evidence from patients with selective thermal lesions and fMRI. Frontiers in Human Neuroscience 9 (2015) 431.

[15] Y. Tian, S. Liang, D. Yao. Attentional orienting and response inhibition: insights from spatial-temporal neuroimaging. Neuroscience bulletin 30(1) (2014) 141-151.

[16] C. Jing, M. Dassarath, Z. Yin, H. Liu, K. Yang, W. Gang. Radiation induced temporal lobe necrosis in patients with nasopharyngeal carcinoma: a review of new avenues in its management. Radiation Oncology 6 (2011) 1-8. 
[17] Y. Cui, B. Liu, M. Song, D.M. Lipnicki, J. Li, S. Xie, Y. Chen, P. Li, L. Lu, L. Lv. Auditory verbal hallucinations are related to cortical thinning in the left middle temporal gyrus of patients with schizophrenia. Psychological Medicine (2017) 1-8.

[18] J. Fan, M. Zhong, J. Gan, W. Liu, C. Niu, H. Liao, H. Zhang, C. Tan, J. Yi, X. Zhu. Spontaneous neural activity in the right superior temporal gyrus and left middle temporal gyrus is associated with insight level in obsessive-compulsive disorder. Journal of Affective Disorders 207 (2017) 203-211.

[19] M.F. Glasser, J.K. Rilling. DTI Tractography of the Human Brain's Language Pathways. Cerebral Cortex 18 (2008) 2471.

[20] Q. Shen, F. Lin, X. Rong, W. Yang, Y. Li, Z. Cai, P. Xu, Y. Xu, Y. Tang. Temporal Cerebral Microbleeds Are Associated With Radiation Necrosis and Cognitive Dysfunction in Patients Treated for Nasopharyngeal Carcinoma. International Journal of Radiation Oncology Biology Physics 94 (2016) 1113.

[21] D. Borsook, R. Veggeberg, N. Erpelding, R. Borra, C. Linnman, R. Burstein, L. Becerra. The Insula: A "Hub of Activity" in Migraine. Neuroscientist A Review Journal Bringing Neurobiology Neurology \& Psychiatry 22 (2016) 632.

[22] T.W. Kjaer, M. Nowak, K.W. Kjaer, A.R. Lou, H.C. Lou. Precuneus-prefrontal activity during awareness of visual verbal stimuli. Consciousness \& Cognition 10 (2001) 356.

[23] H.C. Lou, B. Luber, M. Crupain, J.P. Keenan, M. Nowak, T.W. Kjaer, H.A. Sackeim, S.H. Lisanby. Parietal cortex and representation of the mental Self. Proceedings of the National Academy of Sciences of the United States of America 101 (2004) 6827.

[24] T.W. Kjaer, M. Nowak, H.C. Lou. Reflective self-awareness and conscious states: PET evidence for a common midline parietofrontal core. Neuroimage 17 (2002) 1080-1086.

[25] B.A. Vogt, S. Laureys. Posterior Cingulate, Precuneal \& Retrosplenial Cortices: Cytology \& Components of the Neural Network Correlates of Consciousness. Progress in Brain Research 150 (2005) 205.

[26] D. Dörfel, A. Werner, M. Schaefer, R.V. Kummer, A. Karl. Distinct brain networks in recognition memory share a defined region in the precuneus. European Journal of Neuroscience 47 (2009) 19471959.

[27] B.N. Lundstrom, M. Ingvar, K.M. Petersson. The role of precuneus and left inferior frontal cortex during source memory episodic retrieval. Neuroimage 27 (2005) 824-834.

[28] B.N. Lundstrom, K.J. Petersson, M. Johansson, P. Fransson, M. Ingvar. Isolating the retrieval of imagined pictures during episodic memory: activation of the left precuneus and left prefrontal cortex. Neuroimage 20 (2003) 1934-1943.

[29] N. Wenderoth, F. Debaere, S. Sunaert, S.P. Swinnen. The role of anterior cingulate cortex and precuneus in the coordination of motor behaviour. European Journal of Neuroscience 22 (2005) 235.

[30] Y. Tian, A.B. Chica, P. Xu, D. Yao. Differential consequences of orienting attention in parallel and serial search: An ERP study. Brain Research 1391 (2011) 81.

[31] J.S. Lee, G. Park, M.J. Song, K.H. Choi, S.H. Lee. Early visual processing for low spatial frequency fearful face is correlated with cortical volume in patients with schizophrenia. Neuropsychiatric Disease \& Treatment 12 (2016) 1-14.

[32] O. Collignon, G. Vandewalle, P. Voss, G. Albouy, G. Charbonneau, M. Lassonde, F. Lepore. Functional specialization for auditory-spatial processing in the occipital cortex of congenitally blind humans. Proceedings of the National Academy of Sciences of the United States of America 108 (2011) 44354440.

[33] M. Malekmohammadi, W.J. Elias, N. Pouratian. Human thalamus regulates cortical activity via spatially specific and structurally constrained phase-amplitude coupling. Cerebral Cortex 25 (2015) 1618. 
[34] X. Wu, M. Gu, G. Zhou, X. Xu, M. Wu, H. Huang. Cognitive and neuropsychiatric impairment in cerebral radionecrosis patients after radiotherapy of nasopharyngeal carcinoma. Bmc Neurology 14 (2014) 1-6.

[35] Y. Tang, D. Luo, X. Rong, X. Shi, Y. Peng. Psychological disorders, cognitive dysfunction and quality of life in nasopharyngeal carcinoma patients with radiation-induced brain injury. Plos One 7 (2012) e36529.

[36] K.Y. Hsiao, S.A. Yeh, C.C. Chang, P.C. Tsai, J.M. Wu, J.S. Gau. Cognitive function before and after intensity-modulated radiation therapy in patients with nasopharyngeal carcinoma: a prospective study. International Journal of Radiation Oncology Biology Physics 77 (2010) 722-726.

[37] D.O. Oncology, Q.V. Hospital, Candos, QuatreBornes. Báo cáo khoa học: "Radiation Induced Temporal Lobe Necrosis in Patients with Nasopharyngeal Carcinoma: a Review of New Avenues in Its Management" doc. Gastrointestinal Endoscopy 65 (2007) AB155.

[38] M. Cheung, A.S. Chan, S.C. Law, J.H. Chan, V.K. Tse. Cognitive function of patients with nasopharyngeal carcinoma with and without temporal lobe radionecrosis. Archives of Neurology $\mathbf{5 7}$ (2000) 1347-1352.

[39] A.J. Parkin, N.M. Hunkin. Memory loss following radiotherapy for nasal pharyngeal carcinoma - An unusual presentation of amnesia. British Journal of Clinical Psychology 30 (2011) 349-357.

[40] M.S. Hua, S.T. Chen, L.M. Tang, W.M. Leung. Neuropsychological function in patients with nasopharyngeal carcinoma after radiotherapy. Journal of Clinical \& Experimental Neuropsychology 20 (1998) 684-693. 